\title{
Disordered eating v/s fostering a positive body image in adolescents
}

\begin{abstract}
Eating disorders are severe conditions, an enervating psychiatric illness characterized by fluctuations in eating habits or behaviors to control weight. An early diagnosis of disordered eating is important, especially in adolescents, where its prevalence is quite common. The physical signs and symptoms of disordered eating are related to starvation or inappropriate eating patterns and habits, but the disorder also includes emotional and behavior issues which need to be catered at the earliest. Fostering a positive body image is the most effective way to overcome eating disorders and promote self-satisfaction which helps to make informed and reasonable choices when it comes to eating. Findings suggest that there is a need for early prevention efforts prior to the onset of harmful behavioral patterns, also, prevention and treatment interventions to address the high prevalence of disordered eating throughout adolescence and young adulthood is important.
\end{abstract}

Keywords: disordered eating, anorexia nervosa, bulimia nervosa dieting, body image, adolescents, body shaming
Volume 7 Issue 5 - 2017

\section{Insiya Juzer Diwan}

Nutritionist, Pakistan

Correspondence: Insiya Juzer Diwan, Nutritionist, Pakistan, Email insiyadewan@gmail.com

Received: July 10, 2017 | Published: December 01, 2017

\section{Introduction}

Over concerns with body image and shape may lead to potentially dangerous disordered eating behaviors. ${ }^{1-3}$ Eating disorders -Anorexia nervosa (AN) and bulimia nervosa (BN) are disorders characterized by abnormal patterns of eating behavior and disturbances in attitudes and perceptions toward weight and shape .The etiology of these disorders is presumed to be influenced by developmental, social, and biological processes., ${ }^{4,5}$ People nowadays, consider themselves knowledgeable, as they spend notable amount of time reviewing mutable online information about Nutrition and Health from unreliable sources. Unhealthy dieting and disordered eating behaviors among youth are of concern as they have the potential to adversely affect nutrient intake, mental health status, and long-term health outcomes. ${ }^{6}$ Skipping meals, fasting, eating less, consuming a food substitute and in extreme conditions taking diet pills, purging, use of diuretics and laxatives are commonly adopted methods for weight control. ${ }^{2}$ Study findings indicate that adolescents who engage themselves in disordered eating behavior are at a risk of weight gain and obesity in future. Furthermore, in an analysis it was found that adolescents engaging in dieting and those reporting unhealthy weight control behaviors were at two to three times greater risk of being overweight five years later, as compared to adolescents who did not engage in these behaviors at baseline. ${ }^{4}$ Moreover, eating disorders are more prevalent among adolescent girls. ${ }^{6}$ Body shaming: criticism about a person's body size or shape is the root cause, which leads to dissatisfaction with one's body shape. Social norms, peer and media influences, family dynamics are major contributory factors. ${ }^{2}$

\section{Conclusion}

Variations in body size and shape are normal, always foster a positive body image, focus on your positive body aspects, features and traits, never associate your self-esteem with your body image, be pragmatic, always set realistic weight management goals for yourself ,no magic can help you lose weight overnight. Be confident in your skin because being healthy is more important than being zero sized.
Lose weight but not at the verge of losing yourself. Eating disorders might leave you nutrient deficient, leading to several other health complications. Awareness of your own body image issues and feelings related to your own body satisfaction is crucial. Your goal should be to improve social and emotional well-being and to normalize the eating behaviors. Empower yourself by becoming media literate and challenging unhealthy beliefs you may have regarding weight, shape, and eating.

\section{Acknowledgements}

I would like to thank my family and all the people whose assistance proved to be a milestone in the accomplishment of my end goal.

\section{Conflict of interest}

The author declares no conflict of interest.

\section{References}

1. Swanson SA, Crow SJ, Le Grange D, et al. Prevalence and Correlates of Eating Disorders in Adolescents Results From the National Comorbidity Survey Replication Adolescent Supplement. Arch Gen Psychiatry. 2011;68(7):714-723.

2. Judith E Brown, Janet Isaacs, Bea Krinke, et al. Nutrition through life cycle. 4th ed. USA: Wadsworth Cengage Learning; 2010. p. 397-402.

3. Jamie Stang, Mary T Story. Guidelines for Adolescent Nutrition Services, Center for Leadership, Education and Training in Maternal and Child Nutrition. 2005:158-162.

4. Neumark-Sztainer D, Wall M, Larson NI, et al. Dieting and disordered eating behaviors from adolescence to young adulthood: Findings from a 10-year longitudinal study. J Am Diet Assoc. 2011;111(7):1004-1011.

5. Berrettini W. The Genetics of Eating Disorders. Curr Top Behav Neurosci. 2004;1(3):18-25.

6. Neumark-Sztainer D, Hannan PJ. Weight-Related Behaviors among Adolescent Girls and Boys Results From a National Survey. Arch Pediatr Adolesc Med. 2000;154(6):569-577. 